\title{
Gestión logística en la industria salinera del departamento de La Guajira, Colombia
}

\author{
Danny D. López ${ }^{1}$, Gelvis M. Melo ${ }^{1}$ y Darcy L. Mendoza ${ }^{2}$ \\ (1) Facultad de Ingeniería, Universidad de La Guajira, Km. 5 salida a Maicao, Riohacha - Colombia. \\ (correo-e: dlopezj@uniguajira.edu.co; gelvismanuel@uniguajira.edu.co). \\ (2) Facultad de Ciencias Económicas y Administrativas, Universidad de La Guajira, Km. 5 salida a Maicao, Riohacha - \\ Colombia. (correo-e: dmendoza@uniguajira.edu.co).
}

Recibido Jun. 23, 2020; Aceptado Ago. 20, 2020; Versión final Oct. 17, 2020, Publicado Feb. 2021

\section{Resumen}

El propósito de esta investigación es analizar la gestión logística en la industria salinera en La Guajira, Colombia. Se trata de un estudio descriptivo, no experimental y transversal. La población muestreada la componen 39 ingenieros de empresas mineras que se dedican a la explotación y/o procesamiento de la sal en el departamento de La Guajira. Se empleó la técnica de observación mediante encuesta y se construyó un cuestionario. Se obtuvo como resultado falencias con relación a la aplicación de la gestión logística en la minería de la sal en el departamento de La Guajira, por lo que es importante desarrollar acciones para mejorar su planeación y ejecución. Se concluye que la gestión logística se realiza en forma medianamente efectiva, resultando eficientes las estrategias logísticas examinadas, y reflejándose aceptables la logística interna identificada, los procesos logísticos descritos y la estructura organizacional establecida en el presente estudio.

\section{Logistics management in the salt industry of the state of La Guajira, Colombia}

\begin{abstract}
The purpose of this research study is to assess salt industry logistics management in La Guajira state, Colombia. This is a descriptive, non-experimental, and cross-sectional study. The population sample consists of 39 engineers working at mining companies that exploit or process salt in La Guajira state. The observation survey technique was applied and a survey was constructed. The results show that there are flaws in the application of logistics management in La Guajira's salt mining industry. Therefore, it is important to develop actions to improve planning and execution. It is concluded that logistics management is performed fairly effectively and that the logistics strategies examined are efficient and reflect the internal logistics identified, the logistics processes described, and the organizational structure established in the present study.
\end{abstract}




\section{INTRODUCCIÓN}

A medida que aumenta la urbanización, los niveles de actividades logísticas también se mejoran para satisfacer la demanda de consumo (Sekovski et al., 2012). La gestión logística es un componente importante de la cadena de suministro. Un sistema logístico gestiona el flujo de materiales e información, así como el movimiento y el almacenamiento eficiente de datos, bienes y servicios. El objetivo de un sistema logístico es entregar productos terminados al cliente final con el nivel adecuado de servicio y calidad, con los costos más bajos posibles (Strandhagen et al., 2017). En efecto, en los últimos años, se han realizado mejoras en la eficiencia logística para impulsar la competitividad entre empresas de diferentes tamaños a través de enfoques eficientes para la distribución de carga (Flora et al., 2019). Hoy en día, la gestión logística se ha convertido en la columna vertebral de la economía global, impulsando a las empresas y a los responsables políticos a aumentar su preocupación por el impacto de las actividades logísticas en el medio ambiente (Liu et al., 2018).

Ahora bien, las actividades desarrolladas por el sector logístico tienen impactos tanto positivos como negativos en la sociedad. Como tal, las ganancias económicas y sociales son claramente las contribuciones positivas de estas actividades para muchos países en diferentes regiones del mundo (Rodrigues et al., 2020). Para que las empresas desempeñen un buen papel en la exploración de mercados, es necesario que desarrollen eficientemente la actividad logística y que tengan recursos disponibles en forma de capital de trabajo, inversiones y activos fijos (Lepchak y Voese, 2020). Dentro de este marco, Dimitrov (2005) declara que las empresas deben dominar la gestión logística y su implementación para mejorar sus prácticas, herramientas y métodos de gestión aplicados. Es así como, la gestión logística aún puede ser un obstáculo para las nuevas empresas porque no tienen experiencia, conocimiento y reputación para elegir eficientemente la cadena de suministro menos costosa (Naudé y Matthee, 2011).

Ciertamente, en Colombia este proceso ha representado grandes fusiones, adquisiciones y alianzas estratégicas entre empresas que brindaban pocos servicios de logística, para agrupar un portafolio de servicios más amplio que ofreciera soluciones de gestión logística en toda la cadena de abastecimiento de sus clientes. La estrategia de cooperación entre las empresas nacionales tomó lugar como un esquema de protección y competencia frente a las multinacionales que llegaron después de la apertura económica (González, 2015).

De modo particular, en términos de gestión logística, se puede decir que en la industria salinera en el departamento de La Guajira, los sistemas de gestión logística abarcan la explotación, procesamiento y comercialización de la sal, sin embargo, no cuentan con una organización de las actividades y la mayoría de sus trabajadores no están capacitados y titulados en las labores que desempeñan. También, no poseen sistemas de transporte de alta calidad, tanto de la materia prima como los productos terminados, abarcando todos los procesos de la cadena de abastecimiento, así como el flujo de suministro y el control de inventarios. Los procesos realizados no cumplen a cabalidad con los factores logísticos, puesto que se podrían sugerir mejoras y otros no se implementan, los cuales podrían llevar a un mejor rendimiento y productividad (López, Melo y Mendoza, 2019).

Aunado a lo descrito anteriormente, en el municipio de Manaure (La Guajira), la industria salinera tiene una planeación ineficaz del procesos de explotación de la sal, también, la administración de los recursos minerales es deficiente y el sistema de transporte presenta problemas operativos. En cuanto a los procesos logísticos, el departamento de ventas presenta deficiencias en cuanto a la comercialización internacional por vía marítima y se podría promocionar más el producto, ampliando los mercados. Evidentemente, hay falencias en cuanto a la calidad de los procesos y la gestión de maquinaria y equipos. La competitividad de la industria salinera es baja, de modo que el procesamiento no le permite comercializar un producto de mejores cualidades técnicas (López, Melo y Mendoza, 2019).

De igual forma, en la industria salinera del municipio de Uribia (La Guajira), los trabajadores y los operadores de los cargadores no hacen uso de los elementos de protección personal, por lo que existe un alto riesgo de posibles accidentes. El laboratorio no cuenta con los instrumentos necesarios para hacer las pruebas de calidad de la sal, por lo cual, se ven obligados a solicitar los servicios de un laboratorio externo. Las instalaciones físicas necesitan mantenimiento porque están corroídas. Las señales de seguridad y precauciones dentro del área de trabajo necesitan ubicarse en lugares con mejor visibilidad y un tamaño más grande. Hay aspectos que mejorar en cuanto a la infraestructura, organización, manejo de personal, capacitación del mismo y garantizar su integridad física, de una mejor forma (López, Melo y Mendoza, 2019).

En ese sentido, en el municipio de Maicao (La Guajira), la industria salinera no cuenta con una planificación en cuanto al transporte y recepción de los materiales, con una secuencia de los procesos que allí son realizados. El acopio de materiales para producción no es el correcto, porque las herramientas de trabajo son escasas y oxidadas. Los trabajadores no cuentan con formación técnica profesional, son empíricos y no son 
capacitados, lo que hace que los procesos internos no sean correctos para ofrecer un producto de excelente calidad (López, Melo y Mendoza, 2019).

La realización de esta investigación radica en la importancia que tiene la minería de la sal, como una actividad económica prioritaria de La Guajira que se distingue a nivel nacional por ser líder en producción. Es necesario conocer al detalle las operaciones y actividades que permiten se genere el producto deseado y los problemas que aquejan al sector. Permitirá conocer la realidad de la logística de la sal en la región y su distribución para llegar a todo el país, lo que asiente identificar la situación actual, como se describe el análisis integral de planificación y los principales problemas de desarrollo frente a este tipo de explotación. El propósito de esta investigación es analizar la gestión logística en la industria salinera en La Guajira, Colombia. A continuación se presentan la metodología, los resultados y discusión y las conclusiones.

\section{METODOLOGÍA}

Se realizó un análisis descriptivo, No experimental y transversal (Balnaves \& Caputi, 2001). En particular, la población fue accesible y Censal (Trochim \& Donnelly, 2006); correspondió a los ingenieros de las empresas mineras que se dedican a la explotación y/o procesamiento de la sal en el departamento de La Guajira (Tabla 1). De igual forma, se empleó la técnica de observación mediante encuesta (Hernández et. al., 2010). Para el proceso de medición de las variables del presente estudio se construyó un (1) cuestionario (Tabla 2) con 38 ítems, 2 ítems para cada uno de los 19 indicadores agrupados en las 5 dimensiones de la gestión logística.

Tabla 1: Población: Ingenieros de la industria minera en el departamento de La Guajira

\begin{tabular}{|l|l|c|}
\hline Municipio & Nombre de la empresa salinera & Ingenieros \\
\hline Manaure & Big Group Salinas Colombia S.A.S. & 4 \\
\hline Uribia & Molino y Procesadora Indusalca Ltda & 4 \\
\hline Uribia & Molino y Procesadora Razu Reyna Blanca S.A.S. & 3 \\
\hline Uribia & Molino de Sal Prosalmar & 2 \\
\hline Uribia & Molino de Sal Multisal Ltda & 3 \\
\hline Uribia & Molino de Sal El Guajiro & 2 \\
\hline Uribia & Molino de Sal El Porvenir & 2 \\
\hline Uribia & Molino de Sal La Provincia & 2 \\
\hline Uribia & Molino de Sal Oro Blanco & 2 \\
\hline Uribia & Molino de Sal La Ye & 2 \\
\hline Uribia & Molino De Sal Procosalca Ltda. & 2 \\
\hline Maicao & Molino y Procesadora Disalmar Ltda. & 3 \\
\hline Maicao & Molino y Procesadora Prosainco Ltda. & 2 \\
\hline Maicao & Molino y Procesadora Distrisales Ltda. & 2 \\
\hline Maicao & Molino de Sal Multisales Ltda. & 2 \\
\hline Maicao & Molino Todo Sal S.A.S. & 39 \\
\hline Total & 16 empresas salineras & \\
\hline
\end{tabular}

El cuestionario presenta una escala de actitudes Likert, con preguntas de tipo estimación y por su forma cerrada, conformada por 5 opciones de respuesta (totalmente de acuerdo, de acuerdo, neutral, en desacuerdo, totalmente en desacuerdo), con una ponderación del cinco al uno (5-1), con una escala estadística de medición ordinal (Tabla 3). Posteriormente, se elaboró una matriz para colocar los resultados de los cuestionarios aplicados, seguidamente se tabularon los datos obtenidos, presentándolos en tablas de doble entrada de acuerdo con la variable objeto de estudio y sus respectivas dimensiones e indicadores (Tabla 4). 
Tabla 2. Gestión logística: Dimensiones e indicadores

\begin{tabular}{|l|l|}
\hline Dimensiones & Indicadores \\
\hline $\begin{array}{l}\text { Logística interna. Comprende actividades de logística } \\
\text { dentro de los muros de una organización, por ejemplo, } \\
\text { transportes internos, manejo de materiales, almacenaje } \\
\text { y embalaje (Jonsson, 2008) }\end{array}$ & $\begin{array}{l}\text { Planificación (Corredor, 2004), Recursos materiales } \\
\text { (Sandberg, 2013), Organización por procesos, Formación }\end{array}$ \\
\hline $\begin{array}{l}\text { Logística externa. Son todas las actividades asociadas } \\
\text { con la recopilación, almacenamiento y distribución física } \\
\text { del producto a los compradores, como almacén de } \\
\text { materias terminadas, manejo de materiales, operación y } \\
\text { selección de vehículos de entrega, soporte técnico, } \\
\text { logística inversa, etcétera (Vásquez y Layton, 2013; } \\
\text { González, 2015) }\end{array}$ & \\
\hline $\begin{array}{l}\text { Procesos logísticos. Son el movimiento de los bienes } \\
\text { correctos en la cantidad adecuada hacia el lugar, Distribución física (Ocampo, 2009; } \\
\text { correcto en el momento apropiado, los cuales permiten } \\
\text { la optimización de los recursos de una organización o o } \\
\text { empresa en todas sus áreas de labor (Franklin, 2005) }\end{array}$ & $\begin{array}{l}\text { Planificación de compras, Gestión de pedidos, Análisis de } \\
\text { inventario (Sangri, 2014), Selección de proveedores } \\
\text { (Osorio, Arango y Ruales, 2011) }\end{array}$ \\
\hline $\begin{array}{l}\text { Estructura organizacional. Conjunto de elementos } \\
\text { relativamente estables que se relacionan en el tiempo y } \\
\text { en el espacio para formar una totalidad (Chiavenato, } \\
\text { 2006) }\end{array}$ & $\begin{array}{l}\text { Talento humano, Calidad, Tecnología, Estrategia } \\
\text { organizacional }\end{array}$ \\
\hline $\begin{array}{l}\text { Estrategias logísticas. Son estrategias prácticas de } \\
\text { logística que por lo general comienzan con las metas del } \\
\text { negocio y con los requerimientos de servicio del cliente } \\
\text { (Ballou, 2004) }\end{array}$ & $\begin{array}{l}\text { Competitividad, Plan logístico, Diseño de Procesos (Ballou, } \\
\text { 2004), Sistema logístico }\end{array}$ \\
\hline
\end{tabular}

Tabla 3. Ponderación para los cuestionarios de escala de actitud Likert

\begin{tabular}{|c|c|c|c|c|}
\hline $\begin{array}{c}\text { Totalmente } \\
\text { De Acuerdo }\end{array}$ & De Acuerdo & $\begin{array}{c}\text { Ni De Acuerdo, Ni En } \\
\text { Desacuerdo }\end{array}$ & En Desacuerdo & $\begin{array}{c}\text { Totalmente En } \\
\text { Desacuerdo }\end{array}$ \\
\hline 5 & 4 & 3 & 2 & 1 \\
\hline
\end{tabular}

Tabla 4. Categorías de grados de aplicación de indicadores, dimensiones y gestión logística

\begin{tabular}{|l|l|}
\hline Valores & Categoría \\
\hline $1-2,33$ & Aplica deficientemente \\
\hline $2,34-3,66$ & Aplica medianamente \\
\hline $3,67-5$ & Aplica eficientemente \\
\hline
\end{tabular}

Con el propósito de recabar la información necesaria para lograr medir la variable objeto de estudio, se diseñó un instrumento para la recolección de datos, el cual fue validado mediante el juicio de expertos, este método consiste en impartir el mismo a cinco (5) expertos en las áreas relacionadas con gestión logística y metodología de la investigación, que evalúan y posteriormente formulan sus opiniones en cuanto a la pertinencia de los ítems con los objetivos, variables, indicadores y redacción de los mismos.

Posterior a la validez de los expertos, se aplicó una prueba piloto a veinte (20) Líderes de procesos de las empresas mineras que se dedican a la explotación y/o procesamiento de la sal en el departamento de La Guajira. La confiabilidad se determinó mediante la obtención del Coeficiente de consistencia interna de Alfa Cronbach y sustituyendo la siguiente formula con los valores específicos:

$\alpha=\left[\frac{K}{K-1}\right]\left[1-\frac{\sum_{i=1}^{k} S_{i}^{2}}{S_{t}^{2}}\right]$

Siendo $S_{i}^{2}$ la suma de varianzas de cada ítem, $S_{t}^{2}$ la varianza del total de filas (puntaje total de los jueces) y $K$ el número de preguntas o ítems. De acuerdo con el cálculo de la confiabilidad, se obtuvo un valor de 0,93 lo que aprueba que el instrumento sí es confiable, para la variable gestión logística. 


\section{RESULTADOS Y DISCUSIÓN}

Para la mayoría de los encuestados, la dimensión Logística interna se aplica medianamente, aunque no ocurre lo mismo para los indicadores que componen dicha dimensión: Mientras para la mayoría la planificación y la organización por procesos se aplican eficientemente, los recursos materiales y la formación se aplican deficientemente (este último indicador no se aplica eficientemente para ninguno de los encuestados) (Tabla $5)$.

Tabla 5: Dimensión Logística interna: Distribución grado de aplicación por indicadores

\begin{tabular}{|l|l|l|l|}
\hline \multirow{2}{*}{ Indicadores } & \multicolumn{3}{|c|}{ Aplica } \\
\cline { 2 - 4 } & Deficientemente & Medianamente & Eficientemente \\
\hline Planificación & $2.56 \%$ & $41.03 \%$ & $56.41 \%$ \\
\hline Recursos materiales & $56.41 \%$ & $41.03 \%$ & $2.56 \%$ \\
\hline Organización por procesos & $2.56 \%$ & $33.33 \%$ & $64.10 \%$ \\
\hline Formación & $58.97 \%$ & $41.03 \%$ & $0.00 \%$ \\
\hline Dimensión & $2.56 \%$ & $94.87 \%$ & $2.56 \%$ \\
\hline
\end{tabular}

Para la mayoría de los encuestados, la dimensión Logística externa se aplica deficientemente, lo cual se corrobora con la percepción de deficiente aplicación de los indicadores de transporte y flujo de logística (este último indicador no se aplica eficientemente para ninguno de los encuestados). La distribución física, por su parte, se aplica medianamente para la mayoría de los encuestados, aunque lo hace deficientemente para más de la tercera parte de ellos (Tabla 6).

Tabla 6: Dimensión Logística externa: Distribución grado de aplicación por indicadores

\begin{tabular}{|l|l|l|l|}
\hline \multirow{2}{*}{ Indicadores } & \multicolumn{3}{|c|}{ Aplica } \\
\cline { 2 - 4 } & Deficientemente & Medianamente & Eficientemente \\
\hline Flujo de logística & $61.54 \%$ & $38.46 \%$ & $0.00 \%$ \\
\hline Distribución física & $35.90 \%$ & $46.15 \%$ & $17.95 \%$ \\
\hline Transporte & $76.92 \%$ & $17.95 \%$ & $5.13 \%$ \\
\hline Dimensión & $56.41 \%$ & $43.59 \%$ & $0.00 \%$ \\
\hline
\end{tabular}

Para la mayoría de los encuestados, la dimensión Procesos logísticos se aplica medianamente, aunque solamente ocurre lo mismo para la gestión de pedidos, mientras la planificación de compras se aplica eficientemente para la mayoría de ellos (este indicador no se aplica deficientemente para ninguno de los encuestados) y se aplican deficientemente el análisis de inventario y la selección de proveedores (este último indicador no se aplica eficientemente para ninguno de los encuestados) (Tabla 7).

Tabla 7: Dimensión Procesos logísticos: Distribución grado de aplicación por indicadores

\begin{tabular}{|l|l|l|l|}
\hline \multirow{2}{*}{ Indicadores } & \multicolumn{3}{|c|}{ Aplica } \\
\cline { 2 - 4 } & Deficientemente & Medianamente & Eficientemente \\
\hline Planificación de compras & $0.00 \%$ & $28.21 \%$ & $71.79 \%$ \\
\hline Gestión de pedidos & $20.51 \%$ & $56.41 \%$ & $23.08 \%$ \\
\hline Selección de proveedores & $61.54 \%$ & $38.46 \%$ & $0.00 \%$ \\
\hline Análisis de inventario & $58.97 \%$ & $35.90 \%$ & $5.13 \%$ \\
\hline Dimensión & $15.38 \%$ & $84.62 \%$ & $0.00 \%$ \\
\hline
\end{tabular}

Para la mayoría de los encuestados, la dimensión Estructura organizacional se aplica medianamente, aunque solamente ocurre lo mismo para la tecnología (la cual, sin embargo, se aplica deficientemente para un porcentaje importante de ellos), mientras la calidad se aplica deficientemente para la mayoría de los encuestados y el talento humano y la estrategia organizacional se aplican eficientemente (este último indicador no se aplica deficientemente para ninguno de los encuestados) (Tabla 8). 
Tabla 8: Dimensión Estructura organizacional: Distribución grado de aplicación por indicadores

\begin{tabular}{|l|l|l|l|}
\hline \multirow{2}{*}{ Indicadores } & \multicolumn{3}{|c|}{ Aplica } \\
\cline { 2 - 4 } & Deficientemente & Medianamente & Eficientemente \\
\hline Talento humano & $2.56 \%$ & $38.46 \%$ & $58.97 \%$ \\
\hline Calidad & $56.41 \%$ & $35.90 \%$ & $7.69 \%$ \\
\hline Tecnología & $46.15 \%$ & $48.72 \%$ & $5.13 \%$ \\
\hline Estrategia organizacional & $0.00 \%$ & $33.33 \%$ & $66.67 \%$ \\
\hline Dimensión & $7.69 \%$ & $79.49 \%$ & $12.82 \%$ \\
\hline
\end{tabular}

Para la mayoría de los encuestados, la dimensión Estrategias logísticas se aplica eficientemente, lo cual se corrobora con la percepción de eficiente aplicación de los indicadores de diseño de procesos, sistema logístico y competitividad (este último indicador no se aplica deficientemente para ninguno de los encuestados). El plan logístico, por su parte, se aplica medianamente para la mayoría de los encuestados (Tabla 9).

Tabla 9: Dimensión Estrategias logísticas: Distribución grado de aplicación por indicadores

\begin{tabular}{|l|l|l|l|}
\hline \multirow{2}{*}{ Indicadores } & \multicolumn{3}{|c|}{ Aplica } \\
\cline { 2 - 4 } & Deficientemente & Medianamente & Eficientemente \\
\hline Competitividad & $0.00 \%$ & $28.21 \%$ & $71.79 \%$ \\
\hline Plan logístico & $28.21 \%$ & $56.41 \%$ & $15.38 \%$ \\
\hline Diseño de Procesos & $2.56 \%$ & $17.95 \%$ & $79.49 \%$ \\
\hline Sistema logístico & $5.13 \%$ & $33.33 \%$ & $61.54 \%$ \\
\hline Dimensión & $0.00 \%$ & $48.72 \%$ & $51.28 \%$ \\
\hline
\end{tabular}

Para la totalidad de los encuestados, la Gestión logística se aplica medianamente, lo cual se corrobora con la percepción de mediana aplicación de las dimensiones de logística interna, estructura organizacional y procesos logísticos, mientras la logística externa se aplica deficientemente para la mayoría de los encuestados (las dos últimas dimensiones no se aplican eficientemente para ninguno de ellos) y las estrategias logísticas se aplican eficientemente para la mayoría de ellos (esta dimensión no se aplica deficientemente para ninguno de los encuestados) (Tabla 10).

Tabla 10: Variable Gestión logística: Distribución grado de aplicación por dimensiones

\begin{tabular}{|l|l|l|l|}
\hline \multirow{2}{*}{ Dimensiones } & \multicolumn{3}{|c|}{ Aplica } \\
\cline { 2 - 4 } & Deficientemente & Medianamente & Eficientemente \\
\hline Logística interna & $2.56 \%$ & $94.87 \%$ & $2.56 \%$ \\
\hline Logística externa & $56.41 \%$ & $43.59 \%$ & $0.00 \%$ \\
\hline Procesos logísticos & $15.38 \%$ & $84.62 \%$ & $0.00 \%$ \\
\hline Estructura organizacional & $7.69 \%$ & $79.49 \%$ & $12.82 \%$ \\
\hline Estrategias logísticas & $0.00 \%$ & $48.72 \%$ & $51.28 \%$ \\
\hline Variable & $0.00 \%$ & $100.00 \%$ & $0.00 \%$ \\
\hline
\end{tabular}

En lo referente al objetivo general de la presente investigación, los autores Casanova y Cuatrecasas (2001) argumentan que, la logística es el área encargada del diseño y gestión del flujo de información y de materiales entre clientes y proveedores (distribución, fabricación, aprovisionamiento, almacenaje y transporte) con el objetivo de disponer del material adecuado, en el lugar adecuado, en la cantidad adecuada y en el momento oportuno, al mínimo coste posible y según la cantidad y servicio predefinidos para ofrecer a nuestros clientes; definición que según los resultados de la presente investigación solo aplica medianamente en la industria salinera del departamento de La Guajira.

De la misma forma, los autores Cespón y Auxiliadora (2003) señalan que, la logística es el proceso de gestionar los flujos material e informativo de materias primas, inventario en proceso, productos acabados, servicios y residuales desde el suministrador hasta el cliente, transitando por las etapas de gestión de los aprovisionamientos, producción, distribución física y de los residuales; evidentemente, al tomar en atención los datos adquiridos respecto a las medias de los dimensiones de la variable "Gestión logística", puede deducirse que la mayor debilidad de ésta apunta sobre Logística externa con que se desarrolla la minería de la sal, mientras que la mayor fortaleza viene dada por las Estrategias logísticas de la industria, en el departamento. 
En ese contexto, realizando una comparación con la conclusión de la investigación de García y García (2010), la cual dice que es necesario contar con infraestructura eficiente, integradora y que garantice la competitividad de la producción nacional, necesidad esta que satisface el transporte férreo de calidad, movilizado a través de dobles calzadas férreas, de diseños tecnológicos modernos; se puede argumentar, de acuerdo con los resultados, que la gestión logística de la minería de la sal desarrollada por las empresas en el departamento de La Guajira, debe enfocarse en entregar el producto de forma eficiente a los clientes y/o consumidores, en mejorar la distribución en planta de las maquinarias y equipos para optimizar la producción, con una infraestructura más adecuada y un sistema de transporte eficiente en todas sus operaciones, utilizando rutas adecuadas para la distribución de la sal, a nivel nacional e internacional.

En esa misma línea, se debe mejorar en los aspectos asociados a los ítems de selección de los mejores proveedores en el mercado, estudio de ofertas que presentan los diferentes proveedores para una mayor rentabilidad del negocio, disposición de un inventario que le permita obtener los insumos en el momento adecuado y producción de la cantidad de sal necesaria para surtir a sus clientes. Ello implica, que los resultados obtenidos se comportan de acuerdo a los aportes de Villarreal (2011), quien en su investigación consiguió que la visión y aplicación de la gestión logística tiene diferente percepción dependiendo del puesto dentro de la empresa, que no existe una articulación a lo largo de la cadena de valor y procesos certificados y que no se tiene en la mayoría de los casos la medición de los tiempos de entrega, y rotación de inventarios, en términos de la Gestión logística como una herramienta para crear valor y ventajas competitivas.

En definitiva, dentro del contenido que plantean los resultados de la investigación, aplica medianamente el aporte de Gómez y Acevedo (2007), quienes relacionan la logística con la acción del colectivo laboral dirigida a garantizar las actividades de diseño y dirección de los flujos material, informativo y financiero, desde sus fuentes de origen hasta sus destinos finales, que deben ejecutarse de forma racional y coordinada con el objetivo de proveer al cliente los productos y servicios en la cantidad, calidad, plazos y lugar demandados con elevada competitividad y garantizando la preservación del medio ambiente, por parte de la industria salinera en el departamento de La Guajira.

\section{CONCLUSIONES}

Para la dimensión Logística interna, se requiere que los recursos materiales (adecuada maquinaria para desarrollar sus actividades y uso racional de sus recursos materiales para ser eficientes) y la formación (capacitar a sus trabajadores para producir sal de excelente calidad e identificar las debilidades en sus trabajadores para mejorarlas) se apliquen eficientemente. En cuanto a la dimensión Logística externa, para que esta se aplique eficientemente, se requiere la eficiente aplicación de los indicadores de transporte (contar con un sistema de transporte eficiente en todas sus operaciones y utilizar rutas adecuadas para la distribución de la sal) y flujo de logística (entregar la sal de forma rápida a los consumidores y realizar un control en el proceso de producción de la sal). En lo referente a la dimensión Procesos logísticos, se requiere la eficiente aplicación del análisis de inventario (disponer de un inventario que le permite obtener los insumos en el momento adecuado y producir la cantidad de sal necesaria para surtir a sus clientes) y la selección de proveedores (preocuparse por la selección de los mejores proveedores en el mercado y estudiar ofertas que presentan los diferentes proveedores para una mayor rentabilidad del negocio).

En lo que respecta a la dimensión Estructura organizacional, se requiere la eficiente aplicación de los indicadores de calidad (cumplir con los estándares de calidad necesarios para satisfacer las necesidades de sus clientes y cumplir con las normas que permiten comercializar la sal con las calidades que exige el mercado) y tecnología (contar con las maquinarias óptimas para producir sal de alta calidad y tener un plan de mantenimiento para realizar un proceso productivo excelente). Una limitación de la presente investigación radica en no disponer de información de perfil de los ingenieros entrevistados, en lo referente a formación, año de graduación, antigüedad en el cargo, antigüedad en la empresa, entre otros aspectos, lo cual permitiría indagar si dichas variables inciden en las percepciones con respecto a los ítems sobre los cuales fueron consultados.

\section{REFERENCIAS}

Balnaves, M., y Caputi, P., Introduction to quantitative research methods: An investigative approach, SAGE Publications Ltd., London, Great Britain, (2001)

Ballou, R., Logística. Administración de la cadena de suministro, Prentice Hall, Pearson Educación, México (2004)

Casanovas, A., y Cuatrecasas, L., Logística empresarial, Gestión 2000, Barcelona, España (2001)

Corredor, J., La Planificación. Nuevos enfoques y proposiciones para su aplicación en el siglo XXI. Vadell Hermanos Editores, C.A., Venezuela (2004). 
Cespón, R., y Auxiliadora, M., Administración de la cadena de suministros, Universidad Tecnológica Centroamericana de Honduras - UNITEC, Tegucigalpa, Honduras (2003)

Chiavenato, I., Introducción a la Teoría General de la Administración, 7ạ. Ed, McGraw-Hill, México (2006)

Díaz, D., Transporte y logística en la economía mundial, doi: 10.33776/rem.v0i38.4713, Revista de Economía Mundial, 38, 19-22 (2014)

Dimitrov, P., Logistics in Bulgarian manufacturing companies, doi: 10.1016/j.ijpe.2004.06.019, International Journal of Production Economics, 93-94(8), 207-215 (2005)

Flora, M., Ewbank, H., y Vieira, J.G.V., Framework for urban freight transport analysis in medium-sized cities, doi: 10.1590/2175-3369.011.e20180203, Revista Brasileira de Gestão Urbana, 11(1), (2019)

Franklin, E., Organización de empresas, McGraw-Hill, España (2005)

García, J., y García, A., Logística para el desarrollo integral colombiano, vías para la competitividad: Las dobles calzadas férreas, Ingeniería Investigación y Desarrollo, 10(1), 24-32 (2010)

Gómez, M., y Acevedo J., La logística moderna en la empresa, Editorial Logicuba, La Habana, Cuba (2007)

González, J., Contratación logística en Colombia: implementación de un operador logístico integral, Semestre Económico, 18(38), 215-238 (2015)

Guisao, E., y Zuluaga, A., Distribución física internacional (DFI). Caso de aplicación productos de panela pulverizada Biopanela, Revista Escuela De Administración De Negocios, 70, 157-165 (2011)

Hernández, R., Baptista, P., y Fernández, C., Metodología de la investigación, McGraw-Hill Interamericana, México (2010) Jonsson, P., Logistics and supply chain management, McGraw-Hill, New York, USA (2008)

Lepchak, A., y Voese, S.B., Evaluation of the efficiency of logistics activities using Data Envelopment Analysis (DEA), doi: 10.1590/0104-530x3371-20, Gestão \& Produção, 27(1), (2020)

Liu, J., Yuan, C., Hafeez, M., y Yuan, Q., The relationship between environment and logistics performance: Evidence from Asian countries, doi: 10.1016/j.jclepro.2018.08.310, Journal of Cleaner Production, 204, 282-291 (2018)

López, D., Melo, G., y Mendoza, D., Logística integral de la minería de sal en La Guajira, Colombia, Universidad de La Guajira, La Guajira, Colombia (2019)

Naudé, W., y Matthee, M., The impact of transport costs on new venture internationalization, doi: 10.1007/s10843-0100066-6, Journal of International Entrepreneurship, 9(1), 62-89 (2011)

Ocampo, P., Gerencia logística y global, doi: 10.21158/01208160.n66.2009.477, Revista Escuela De Administración De Negocios, 66, 113-136 (2009)

Osorio, J., Arango, D., y Ruales, C., Selección de proveedores usando el despliegue de la función de calidad difusa, Revista EIA, 8(15), 73-83 (2011)

Rodrigues, H.S., Alves, W., y Silva, Â., The impact of lean and green practices on logistics performance: A structural equation modelling, doi: 10.1590/0103-6513.20190072, Production, 30(1), (2020)

Sandberg, E., Understanding logistics based competition in retail-a business model approach, doi: 10.1108/09590551311306237, International Journal of Retail \& Distribution Management, 41(3), 176-188 (2013)

Sangri, A, Administración de compras, Editorial Patria S.A., México (2014)

Sekovski, I., Newton, A., y Dennison, W.C., Megacities in the coastal zone: using a driver-pressure-stateimpact-response framework to address complex environmental problems, doi: 10.1016/j.ecss.2011.07.011, Estuarine, Coastal and Shelf Science, 96(1), 48-59 (2012)

Strandhagen, J. O., Vallandingham, L. R., y otros cuatro autores, Logistics 4.0 and emerging sustainable business models, doi: 10.1007/s40436-017-0198-1, Advances in Manufacturing, 5(4), 359-369 (2017)

Trochim, W., y Donnelly, J., The research methods knowledge base, 3rd Edition, Cengage Learning, Ohio, United States, (2006)

Vásquez, J., y Layton, P., Modelo del proceso de logística externa de las empresas localizadas en el municipio de Funza - Cundinamarca, doi: 10.31910/rudca.v16.n2.2013.933, Revista U.D.C.A Actualidad \& Divulgación Científica, 16(2), 563$570(2013)$

Villarreal, F., Logística integral, una herramienta para crear valor y ventajas competitivas, Revista Científica Teorías, Enfoques y Aplicaciones en las Ciencias Sociales, 3(6), 99-116 (2011) 